Artigo Original

Original Article

\title{
Efeito da Eutonia no Tratamento da Fibromialgia
}

\section{Eutonia Effect in the Fibromyalgia Treatment}

\author{
Cecília Maeda $^{(1)}$, José Eduardo Martinez ${ }^{(2)}$, Matilde Neder $^{(3)}$
}

\section{RESUMO}

Objetivo: avaliar a eutonia como método terapêutico auxiliar no tratamento da fibromialgia (FM). Métodos: estudou-se um grupo de 20 mulheres, segundo os critérios do American College of Rheumatology (ACR), com duração mínima de seis meses, sem alterações no tratamento medicamentoso ou prescrição de exercícios nesse período. As pacientes foram avaliadas através do questionário de dados sociodemográficos; Escala Visual Analógica (EVA) para dor e questionário genérico de avaliação da qualidade de vida (medical outcomes study 36 - item short-form health survey - SF-36). As avaliações foram realizadas imediatamente antes e após as sessões e ainda um ano após o término das sessões. As pacientes foram submetidas a 25 sessões de eutonia acompanhadas de educação em saúde. Resultados: houve melhora significativa na intensidade da dor, de acordo com a EVA (7,82 antes e 4,17 após) e em todos os domínios do SF-36 na avaliação imediatamente após a eutonia. Os resultados da dor se mantiveram um ano após a intervenção $(4,18)$. Em relação às escalas do SF-36, a melhora também se manteve, porém com intensidade menor do que no resultado imediato.Não há qualquer modalidade terapêutica isolada que atenda todas as demandas da FM. A utilização de técnicas psicológicas tem sido proposta como complementação à terapia medicamentosa e aos exercícios físicos. A eutonia é uma técnica que enfatiza o autoconhecimento e, portanto, poderia ser útil na abordagem da FM. Os resultados desse estudo mostraram efeito positivo na intensidade da dor e na qualidade de vida dos pacientes. Conclusão: a eutonia é útil como método terapêutico na FM associado ao tratamento habitual dos pacientes estudados. Seu efeito é particularmente importante na intensidade da dor difusa crônica e na qualidade de vida.

Palavras-chave: fibromialgia, eutonia, qualidade de vida, dor.

\begin{abstract}
Objective: To evaluate the usefulness eutonia for the treatment fibromyalgia. Methods: It was studied, for a six month period, 20 women diagnosed by the American College of Rheumatology (ACR) Classification Criteria. There was no change on pharmacological treatment or exercise prescription. They were evaluated immediately before and after eutonia sessions and one year after this treatment. The tools used were: socio-demographic questionnaire, Visual Analogic Scale (VAS) for pain, The Medical Outcome Study 36 Short Form Survey (SF-36). The patients were submitted to 25 sessions of eutonia technique and health education. Results: There was a significant improvement in pain intensity by VAS (7,82 before and 4,17 after the intervention) and all SF-36 scales at the evaluation just after treatment. The improvement on pain was maintained after one year (4,18). The SF-36 scales results persisted until a year after intervention, although with a smaller intensity than the ones at the final of intervention. There is no therapeutic modality that can in an isolated manner solve all fibromyalgia patients demands. The psychological techniques combined to medicines and exercises have been shown to be useful in fibromyalgia patients' approach. Eutonia is a psychological technique that emphasizes self-knowledge as a therapy method and could be useful in the fibromyalgia approach. This study results show that eutonia causes a positive effect on pain and quality of life. Conclusion: Eutonia is a psychological technique useful as a treatment for fibromyalgia. In this study it was combined to usual treatment. Its effect is particularly important at pain intensity and quality of life.
\end{abstract}

Keywords: fibromyalgia, eutonia, quality of life, pain.

Pontifícia Universidade Católica de São Paulo (PUC-SP). Recebido em 04/08/05. Aprovado, após revisão, em 14/1 1/05.

1. Doutora em Psicologia Clínica,Professora do Curso de Psicologia Hospitalar da PUC-SP.

2. Titular do Departamento de Medicina da PUC-SP.

3. Titular do Departamento de Psicologia Clínica, Programa de Pós-Graduação em Psicologia Clínica: Núcleo de Psicossomática e Psicologia Hospitalar da PUC-SP.

Endereço para correspondência: José Eduardo Martinez, Rua Riachuelo, 460, s.103, Sorocaba, CEP 18035-330, SP, Brasil. 


\section{INTRODUÇÃO}

A fibromialgia (FM) é uma síndrome caracterizada por dor generalizada e hipersensibilidade à palpação em locais anatômicos definidos, denominados pontos dolorosos (tender points). Acomete principalmente mulheres entre 30 e 60 anos; apesar de pesquisas recentes demonstrarem sua ocorrência em homens e crianças. ${ }^{(1,2)}$

O diagnóstico é essencialmente clínico. Para efeitos de classificação, segundo o American College of Rheumatology (ACR), é necessária a presença de dor difusa por um prazo superior a três meses consecutivos e, no mínimo, em 11 dos 18 pontos dolorosos. São observadas ainda alterações do sono, como despertares noturnos freqüentes, agitação noturna e acordar precoce. Outras queixas referem-se a cefaléias crônicas, parestesias, rigidez articular, sensibilidade ao frio, sensação de edema, perturbações da memória e concentração, fadiga generalizada, além de co-morbidades, como a depressão e a ansiedade. ${ }^{(1,2)}$

Fatores psicossociais também influenciam na gravidade dos sintomas que, num mecanismo de círculo vicioso, geram incapacidade funcional e alterações psicológicas e afetivas em graus variáveis. ${ }^{(3,4)}$

A abordagem psicológica é fundamental para avaliar a personalidade e o estado emocional dos pacientes, visto que a FM leva a restrições de movimentos físicos e com um nível de dor constante, gerando freqüentemente reverberações socioeconômicas e profissionais importantes. $\mathrm{O}$ tratamento psicológico auxilia a buscar recursos internos e planejar estratégias de enfrentamento, pois corpo e mente atuam de forma interdependente e interativa. ${ }^{(5-11)}$

Nessa categoria também foram reunidos alguns métodos de integração psicofísica que utilizam, entre outros, o relaxamento, a meditação, os trabalhos de consciência corporal e o biofeedback. Por serem instrumentos utilizados como auxiliares e/ou complementares em várias abordagens psicoterapêuticas, podem ser utilizados também em programas educativos, que visem uma abrangência mais ampla do controle dos sintomas e co-morbidades decorrentes da FM, e em programas de orientação multidisciplinar.

Turner e Romano ${ }^{(6)}$ destacam que as técnicas de relaxamento, além dos benefícios efetivos que trazem, também podem ser consideradas um recurso para levar o paciente a desviar sua atenção da dor.

Souza ${ }^{(7)}$ avaliou o efeito de técnicas de relaxamento sobre a dor, durante oito sessões ( 2 meses) em 60 mulheres com fibromialgia, distribuídas em três grupos de 20 pacientes. Um grupo foi submetido à técnica denominada visualização dirigida; outro utilizou o treino do relaxamento progressivo de Jacobson; e o terceiro grupo comparativo utilizou apenas medicação. Como instrumentos de avaliação foram utilizados a Escala Visual Analógica (EVA) para dor, o questionário de impacto de FM (Fibromyalgia Impact Questionnaire - FIQ) e o inventário de qualidade de vida (IQV). Os grupos de visualização e relaxamento apresentaram diferenças estatisticamente significativas na intensidade da dor após cada sessão de relaxamento. Por outro lado, não ocorreram diferenças significativas na comparação das duas técnicas entre si, o que permite dizer que ambas atuam de forma semelhante. Os resultados do FIQ se apresentaram melhores na técnica de Jacobson do que na de visualização, e o grupo não tratado melhorou, de forma significativa, a intensidade da dor, como forma de controle das expectativas criadas pela participação na pesquisa.

Outros pesquisadores têm realizado estudos sob o enfoque psicológico no tratamento da FM. Entre as diferentes abordagens psicoterapêuticas, a linha cognitivo-comportamental é utilizada com mais frequiência até o momento.

Os estudos de Nielson et a $\mathrm{I}^{(8)}$ avaliaram a eficácia dessa abordagem em 25 pessoas, que passaram por um programa que envolvia técnicas cognitivas, relaxamento, exercícios aeróbicos e de alongamento, diário de atividades cotidianas, apoio e educação familiar. Neste programa foram mensuradas dimensões objetivas como a gravidade da dor, comportamento da dor e sua influência na vida do paciente, além de outras variáveis como, por exemplo, suporte social, atividade física e relação conjugal. Os resultados finais mostraram mudanças significativas e consistentes nas variáveis objetivas $^{(8)}$.

Keel et al ${ }^{9)}$ compararam os resultados de um grupo experimental (GE) de 14 pacientes com fibromialgia, que passaram pela abordagem cognitivo-comportamental com um grupo controle de 13 pacientes, que passaram apenas por técnicas de relaxamento. $\mathrm{O}$ trabalho do GE aconteceu em 15 sessões semanais de 2 horas, envolvendo informações, instrução de estratégias de autocontrole, discussão em grupo, ginástica e relaxamento. O programa de controle consistia no treinamento autógeno de Schultz. Para os dois grupos foram calculados índices de atividade e de repouso, dor, distúrbios do sono, medicamentos utilizados e uso de fisioterapia. Concluiu-se que o programa cognitivo-comportamental conduziu a mudanças mais efetivas e duradouras do que o treino de relaxamento isolado, supondo-se assim que as orientações informativas e de promoção do autocontrole tenham sido os elementos mais relevantes do programa do GE. 
Portnoi ${ }^{(10)}$ desenvolveu uma pesquisa no Hospital das Clínicas da Faculdade de Medicina da Universidade de São Paulo (FMUSP) com psicoterapia cognitivo-comportamental para pessoas com FM, trabalhando com 12 mulheres que participaram de grupo operativo (Pichon-Riviére) e 11 mulheres no grupo controle. Verificou que as participantes do grupo operativo passaram a utilizar as sensações dolorosas como referência para desenvolver estratégias de enfrentamento das dificuldades decorrentes da FM, ao passo que as melhoras que aconteceram no grupo- controle podem ter ocorrido pelas expectativas geradas pelos períodos de espera. Concluiu ainda que o número de sessões foi insuficiente para o aprofundamento dos temas emergentes das sessões.

Brasio e cols ${ }^{(11)}$, em estudo comparativo entre três técnicas de intervenção psicológica para tratamento da FM - treino de controle de estresse, relaxamento progressivo e reestruturação cognitiva -, pesquisaram 21 pacientes do sexo feminino do interior de São Paulo, divididos em três grupos: Grupo de treino de controle de estresse, Grupo de relaxamento progressivo e Grupo de reestruturação cognitiva. As pacientes foram avaliadas por meio do inventário de sintomas de estresse, escala de ansiedade e depressão, levantamento dos níveis de inassertividade, crenças irracionais e EVA, concluindo que as três técnicas possibilitaram a redução do nível de estresse, a diminuição da ansiedade e depressão e o desenvolvimento da assertividade, sendo que nenhuma das técnicas mostrou-se significativa na redução da percepção das dores. Como conclusão, confirmou-se as controvérsias quanto à origem da FM, destacando-se a necessidade da realização de novos trabalhos com outros tipos de intervenção.

Ainda no âmbito das técnicas psicológicas, a eutonia propõe uma investigação constante do próprio corpo, veículo de aprendizagem e autoconhecimento. O indivíduo tem a possibilidade de poder participar também de sua saúde, como co-responsável pela melhoria dos seus sintomas e do seu bem-estar. ${ }^{(12,13,14)}$

A eutonia é uma técnica de consciência corporal que tem como objetivo o autoconhecimento e o desenvolvimento do senso de percepção, através das possibilidades de conhecimento do funcionamento do corpo, padrões habituais de postura, de movimento, modo de distribuição das tensões, alinhamento e/ou desvios do eixo físico, orientando a construção de um centro de equilíbrio, para que o indivíduo possa ter a possibilidade de se relacionar melhor consigo mesmo e com o mundo.

Utiliza-se de pesquisas no atlas de anatomia, toques exploratórios na pele, na estrutura óssea e muscular e movimentos eutônicos. As sessões são realizadas com materiais auxiliares como bolinhas, bambus, argila, pequenas almofadas, bexigas, sementes, entre outros, visando a ampliação da consciência corporal. Recursos audiovisuais (filmes, diapositivos, músicas) também são utilizados com o objetivo de esclarecer e informar o paciente sobre o funcionamento do próprio corpo.

Considerando que a pedagogia da eutonia se destaca pela prioridade da vivência, pode-se utilizá-la para propiciar a capacidade de inserção do indivíduo no seu meio, melhorando suas condições pessoais, visto que o "estar no mundo" está inserido na própria idéia da eutonia. ${ }^{(12,13,14)}$

Poderá a eutonia ser o instrumento de transformação, favorecendo ao portador de FM a condição de ser ainda responsável por si mesmo? A partir do momento em que a pessoa reconhece e se apropria da necessidade de profundas transformações na sua maneira de ser, seus sintomas podem ganhar novos significados e a doença pode ser vista como uma oportunidade de crescimento. ${ }^{(12,13,14)}$

Se favoráveis, qual é o impacto dessas transformações na qualidade de vida do indivíduo com FM? O papel desta proposta de trabalho é que, através das respostas desse estudo, seja possível alterar a abordagem de pacientes com FM de forma que esta não fique restrita a aspectos científicos. O objetivo é que se estenda ao âmbito social, como possibilidade de modificações nas relações pessoais e familiares, podendo vir a alterar os padrões da existência desse indivíduo, o seu modo de ser no mundo, consigo mesmo e com os outros indivíduos. Sob esse prisma, foram estabelecidos os objetivos desta pesquisa.

1) Avaliar o efeito da eutonia na FM, através dos seguintes parâmetros: intensidade da dor e qualidade de vida.

2) Verificar se os resultados obtidos com a intervenção terapêutica da eutonia se mantiveram um ano após o término da pesquisa.

\section{PACIENTES E MÉTODOS}

\section{CRITÉRIO DE INCLUSÃO}

O grupo de estudo foi composto por mulheres em acompanhamento no ambulatório de reumatologia da Faculdade de Ciências Médicas de Sorocaba da Pontifícia Universidade Católica de São Paulo (PUC-SP), diagnosticadas com FM, através dos critérios de classificação de FM do ACR. ${ }^{(2)}$ 


\section{CRITÉRIOS DE EXCLUSÃO}

As pacientes que não completaram, no mínimo, o ensino fundamental, foram excluídas, assim como pacientes que apresentaram doenças neurológicas e/ou psiquiátricas graves ou grandes dificuldades de locomoção e movimentos.

O grupo foi formado por 20 mulheres. Todas as participantes faziam uso de antidepressivos tricíclicos e analgésicos de acordo com a dor. A medicação permaneceu estável durante todo o tempo do estudo.

\section{INSTRUMENTOS DE AVALIAÇÃO}

1 - Questionário de dados sociodemográficos;

2 - EVA para dor: escala unidimensional utilizada para mensurar a intensidade da dor. Apresenta-se como uma linha impressa, medindo $10 \mathrm{~cm}$ de comprimento e contém em um dos extremos a expressão "sem dor" e na extremidade oposta a expressão "pior dor possível”.(15)

3 - Questionário SF-36: versão validada para o Brasil do questionário genérico de avaliação de qualidade de vida. É composto de oito escalas: capacidade funcional, aspectos físicos, dor, estado geral da saúde, saúde mental, aspectos emocionais, aspectos sociais e vitalidade. Foram consideradas as diferenças entre as médias obtidas nos resultados de cada escala antes, imediatamente após a intervenção terapêutica, e um ano após o término da terapêutica proposta. ${ }^{(16)}$

\section{INTERVENÇÃO TERAPÊUTICA: EUTONIA}

A pesquisa foi realizada nas instalações da Faculdade de Ciências Médicas da Pontifícia Universidade Católica (PUC), localizada em Sorocaba (SP). Foram realizadas 25 sessões segundo o esquema a seguir:

Os três primeiros encontros foram reservados para a aplicação individual dos seguintes questionários e escalas: questionário de dados sociodemográficos; EVA; questionário SF-36.

Do $4^{\circ}$ ao $23^{\circ}$ encontro, foi aplicada a técnica da eutonia, com aulas teóricas e vivências eutônicas, enfocando a reconstrução das percepções conscientes dos diversos segmentos corporais e do corpo em sua globalidade, considerando a postura, a fluidez de movimento, a flexibilidade articular e a expressividade verbal do grupo no relato da própria experiência. Os encontros foram semanais, com duração de duas horas cada, orientados pelo roteiro a seguir, respeitando o ritmo e a necessidade do grupo:

a) Momentos da roda aberta, com relatos sobre a experiência da semana anterior, relativos à percepção corporal, dor, imagens, conexões com conteúdos psíquicos e sentimentos; b) Apresentação de filmes sobre o corpo humano $\left(2^{\circ}\right.$ e $5^{\circ}$ encontros) e sobre FM ( $6^{\circ}$ e $19^{\circ}$ encontros $)$, com recursos audiovisuais informativos;

c) Utilização de música $\left(5^{\circ}, 10^{\circ}, 13^{\circ}\right.$ e $18^{\circ}$ encontros), com o intuito de experimentar movimentos livres e espontâneos, exercitando a capacidade de criar através do próprio corpo e do movimento;

d) Explanações sucintas de anatomia e fisiologia com o atlas anatômico e peças do esqueleto humano, na medida do grau de compreensão do grupo;

e) Pesquisa do segmento corporal a ser trabalhado durante o encontro e vivências de sensopercepção, utilizando materiais auxiliares como bolinhas, sementes, bambus, bexigas, entre outros;

f) Momentos da roda aberta, em que as integrantes são convidadas a participar com comentários sobre a experiência vivida durante a aula, quanto aos aspectos sensoperceptivos e emocionais.

Durante os $24^{\circ}$ e $25^{\circ}$ encontros, após a intervenção terapêutica, foram aplicados individualmente a EVA e o questionário SF-36.

Um ano após o término da pesquisa foi realizado novo encontro para aplicação dos instrumentos de avaliação (T2). Durante esse ano, as pacientes permaneceram em tratamento médico tradicional.

\section{$\overline{\text { ANÁLISE ESTATÍSTICA }}$}

Os procedimentos estatísticos utilizados na análise dos dados foram:

- Descrição da freqüência das variáveis estudadas, relacionada ao resultado do levantamento dos dados sociodemográficos.

- Utilização do Teste T-Pareado para amostras dependentes na análise da EVA e do questionário SF-36.

- A significância estatística foi considerada para $\mathrm{p}<0,05$.

$\mathrm{O}$ projeto da pesquisa, bem como o consentimento pós-informado foram aprovados pela Comissão de Ética em Pesquisa da PUC-SP.

\section{RESULTADOS}

A Tabela 1 mostra os principais dados demográficos das pacientes estudadas. Nossa casuística foi constituída por 20 mulheres com idade média de 45,4 anos, em sua maioria casada, tendo cursado o ensino fundamental.

A Tabela 2 mostra os resultados da variação da intensidade da dor, através da para dor EVA. 
TABELA 1

DADOS DEMOGRÁFICOS DAS PACIENTES PARTICIPANTES ESTUDADAS

\begin{tabular}{ll}
\hline Número & 20 \\
Idade média (intervalo) & $45,4(32-56)$ \\
\hline ESTAD0 CIVIL & \\
\hline Casada & $16(80,0 \%)$ \\
\hline Viúva & $01(5,0 \%)$ \\
Separada & $03(15,0 \%)$ \\
\hline ESCOLARIDADE & \\
\hline Ensino Fundamental & $17(85,0 \%)$ \\
\hline Ensino Médio & $02(10,0 \%)$ \\
Ensino Superior & $01(5,0 \%)$ \\
\hline Duração média em anos (intervalo) & $3,4(2-10)$ \\
\hline
\end{tabular}

TABELA 2

RESUlTAdos DA VARIAČ̃̃ DA INTENSIDADE DA DOR ANTES, IMEDIATAMENTE APÓS E UM ANO APÓS A INTERVENÇÃO COM EUTONIA

\begin{tabular}{|c|c|c|c|c|c|c|}
\hline \multirow[b]{2}{*}{ EVA } & \multicolumn{3}{|c|}{ Diferenças Pareadas } & \multirow[b]{2}{*}{$\mathbf{T}$} & \multirow[b]{2}{*}{$\begin{array}{c}\text { Grau de } \\
\text { liberdade }\end{array}$} & \multirow[b]{2}{*}{$\mathrm{p}$} \\
\hline & Média & $\begin{array}{l}\text { Desvio } \\
\text { Padrão }\end{array}$ & $\begin{array}{l}\text { Erro } \\
\text { Padrão }\end{array}$ & & & \\
\hline T1 - T0 & $-3,647$ & 2,1683 & 0,4974 & $-7,332$ & 18 & 0,0001 \\
\hline $\mathrm{T} 2-\mathrm{T} 1$ & 0,039 & 2,2932 & 0,5405 &,- 072 & 17 & 0,472 \\
\hline T2 - T0 & $-3,672$ & 3,0111 & 0,7097 & $-5,174$ & 17 & 0,0001 \\
\hline
\end{tabular}

Os resultados da avaliação inicial do grupo atingiram a média de 7,83 , e após as sessões de eutonia, a média alterou-se para 4,17. A diferença é estatisticamente significante $(\mathrm{p}<0,001)$, indicando que a eutonia modificou positivamente a percepção da intensidade da dor. Na terceira aplicação da escala, um ano após, o resultado da média foi 4,18 , sendo semelhante a anterior, revelando que após um ano da intervenção a percepção da dor se manteve.

\section{GRÁFICO 1}

AvaliaÇão DA DOR, ATRAVÉS DA ESCALA VISUAL ANALÓGICA (EVA) DE DOR, ANTES, IMEDIATAMENTE APÓS E UM ANO APÓS A EUTONIA

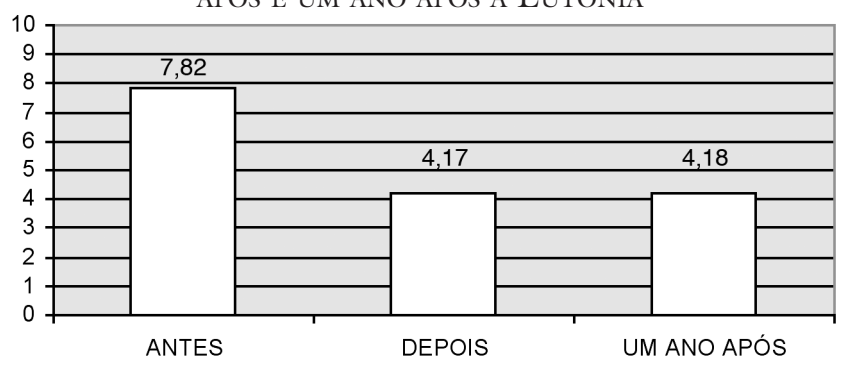

\section{QUESTIONÁRIO SF-36 DE QUALIDADE DE VIDA}

A Tabela 3 mostra os resultados do SF-36 antes e depois da aplicação da eutonia ( $\mathrm{Tl}$ - T0).

\section{TABELA 3}

REsultados DA VARIAÇÃo DA QUALIDADE DE VIDA, ATRAVÉS DAS ESCALAS DO SF-36 ANTES, IMEDIATAMENTE APÓS A INTERVENÇÃO COM EUTONIA (T1 - T0)

\begin{tabular}{c|c|c|c|c|c|c}
\hline & Média & $\begin{array}{c}\text { Desvio } \\
\text { Padrão }\end{array}$ & $\begin{array}{c}\text { Erro } \\
\text { Padrão }\end{array}$ & $\mathbf{T}$ & $\begin{array}{c}\text { Grau de } \\
\text { Liberdade }\end{array}$ & $\mathbf{p}$ \\
\hline $\begin{array}{c}\text { Capacidade } \\
\text { funcional }\end{array}$ & 39,74 & 18,293 & 4,197 & $-9,468$ & 18 & 0,0001 \\
$\begin{array}{c}\text { Aspectos } \\
\text { Físicos }\end{array}$ & 61,84 & 48,138 & 11,044 & $-5,600$ & 18 & 0,0001 \\
\hline Dor & 24,74 & 19,255 & 4,417 & $-5,600$ & 18 & 0,0001 \\
$\begin{array}{c}\text { Geral de } \\
\text { Saúde }\end{array}$ & 31,84 & 12,933 & 2,967 & $-10,732$ & 18 & 0,0001 \\
$\begin{array}{c}\text { Vitalidade } \\
\text { ind }\end{array}$ & 3,42 & 10,007 & 2,296 & $-1,490$ & 18 & 0,077 \\
$\begin{array}{c}\text { Aspectos } \\
\text { Sociais }\end{array}$ & 25,66 & 30,469 & 6,990 & $-3,671$ & 18 & 0,001 \\
\hline $\begin{array}{c}\text { Aspectos } \\
\text { emocionais }\end{array}$ & 68,42 & 37,636 & 8,634 & $-7,924$ & 18 & 0,0001 \\
$\begin{array}{c}\text { Saúde } \\
\text { Mental }\end{array}$ & 5,68 & 13,221 & 3,033 & $-1,874$ & 18 & 0,038 \\
\hline
\end{tabular}

Observa-se que, para os domínios capacidade funcional, aspectos físicos, dor, estado geral da saúde, saúde mental, aspectos emocionais e aspectos sociais, a análise da variância mostrou diferença estatisticamente significante $(\mathrm{p}<0,001)$ entre o momento inicial e após a aplicação da eutonia. $\mathrm{O}$ domínio vitalidade não foi estatisticamente significativo.

\section{GrÁFICO 2}

COMPARAÇÃo dos RESUlTAdos dAS MÉdias dos OITO DOMÍNIOS DO SF-36, CONSIDERANDO TRÊS MOMENTOS: ANTES E DEPOIS DOS ENCONTROS DE EUTONIA, E UM ANO APÓS O TÉRMINO

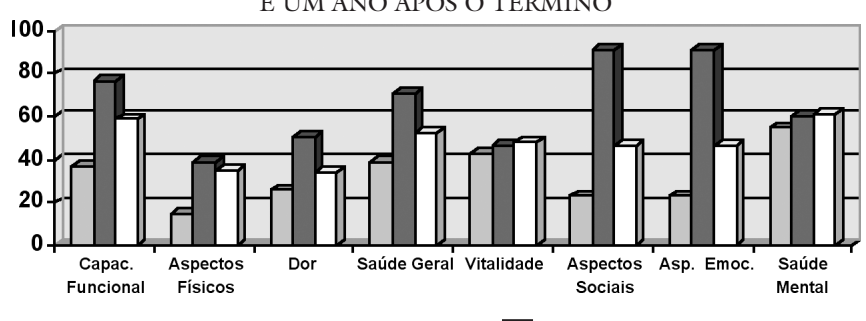


A Tabela 4 mostra os resultados do questionário SF-36 de qualidade de vida, comparando os resultados obtidos antes da intervenção terapêutica da eutonia e um ano após o término da pesquisa (T2 - T0).

Esta tabela demonstra que, em todos os domínios, as diferenças foram estatisticamente significativas, quando comparadas aos resultados da primeira aplicação dos questionários (antes da intervenção) com o segundo momento (depois). Esse fato indica que mesmo após um ano da aplicação da eutonia, a representação da qualidade de vida melhorou em comparação com a primeira aplicação, como se observa nos gráficos a seguir.

\section{TABELA 4}

RESUlTADOS DA VARIAÇÃO DA QUALIDADE DE VIDA, ATRAVÉS DAS ESCALAS DO SF-36, ANTES E UM ANO APÓS A INTERVENÇÃO COM EUTONIA (T2 - T0)

\begin{tabular}{|c|c|c|c|c|c|c|}
\hline & Média & $\begin{array}{l}\text { Desvio } \\
\text { Padrão }\end{array}$ & $\begin{array}{c}\text { Erro } \\
\text { Padrão }\end{array}$ & $\mathbf{T}$ & $\begin{array}{c}\text { Grau de } \\
\text { Liberdade }\end{array}$ & $\mathrm{p}$ \\
\hline $\begin{array}{l}\text { Capacidade } \\
\text { funcional }\end{array}$ & 21,67 & 20,722 & 4,884 & 4,436 & 17 & 0,0001 \\
\hline $\begin{array}{l}\text { Aspectos } \\
\text { Físicos }\end{array}$ & 19,44 & 36,938 & 8,706 & 2,233 & 17 & 0,019 \\
\hline Dor & 7,78 & 14,775 & 3,483 & 2,233 & 17 & 0,019 \\
\hline $\begin{array}{l}\text { Geral de } \\
\text { Saúde }\end{array}$ & 13,06 & 17,751 & 4,184 & 3,120 & 17 & 0,003 \\
\hline Vitalidade & 4,72 & 10,775 & 2,540 & 1,859 & 17 & 0,040 \\
\hline $\begin{array}{l}\text { Aspectos } \\
\text { Sociais }\end{array}$ & 13,89 & 26,040 & 6,138 & 2,263 & 17 & 0,019 \\
\hline $\begin{array}{l}\text { Aspectos } \\
\text { emocionais }\end{array}$ & 22,22 & 52,394 & 13,349 & 1,799 & 17 & 0,045 \\
\hline $\begin{array}{l}\text { Saúde } \\
\text { Mental }\end{array}$ & 6,44 & 13,452 & 3,171 & 2,032 & 17 & 0,029 \\
\hline
\end{tabular}

\section{DISCUSSÃO}

O tratamento da FM se baseia no tripé medicamento, exercício físico e educação em saúde. A associação de técnicas psicológicas com exercícios e medidas educativas poderia potencializar o benefício dessas modalidades terapêuticas. A maior dificuldade para a sua utilização ainda é o acesso, que se mantém restrito àqueles pacientes com maior poder aquisitivo e moradores dos grandes centros. $\mathrm{O}$ trabalho psicológico em grupo é uma tentativa de maximizar o efeito e aumentar o acesso. . $^{(3,17,18,19)}$

O objetivo de nosso estudo foi avaliar a eutonia como umas das técnicas psicológicas que tem em sua essência um caráter pedagógico. O exercício físico não foi avaliado como uma variável a ser estudada, porém sempre estimulamos nossos pacientes a realizá-los regularmente. Foi solicitado ao grupo de estudo que mantivesse a atividade física habitual e evitasse modificações em seus hábitos durante o período de estudo, bem como modificações da terapia medicamentosa.

O racional de nossa proposta baseia-se no papel que a literatura de saúde estabelece para as técnicas psicológicas, em especial àquelas executadas em grupo.

Para Gentry e Owens ${ }^{(20)}$, as psicoterapias em grupo propiciam três benefícios, a saber: a) oportunidade de o paciente conviver com outras pessoas em situação semelhante, além da possibilidade de criar referenciais grupais que diminuam sentimentos de isolamento e alienação; b) permitir ao profissional atuar diretamente em comportamentos que ocorrem no contexto social do grupo, ao mesmo tempo em que compartilha temas referentes à sua evolução clínica e à prevenção de dependência terapêutica; c) representam um recurso mais econômico às instituições, pois propiciam a um maior número de indivíduos tratamento simultâneo com o mesmo profissional.

Dentre as abordagens psicoterapêuticas para o paciente com dor crônica, várias linhas mostram-se úteis. A abordagem comportamental/cognitiva compreende a dor como uma experiência que ocorre em um contexto de relacionamento e social. Esse modelo ressalta que as percepções e crenças de uma pessoa sobre a possibilidade de controlar os efeitos de uma doença influem sobre a característica dos sintomas, a funcionalidade e a capacidade adaptativa ${ }^{(21,22)}$.

A terapêutica psicossomática é outra abordagem utilizada. Ela analisa o paciente como um ser emocionalmente vulnerável, socialmente discriminado e com sua credibilidade posta à prova, impotente diante dos limites que a doença impõe, além de outras facetas que se apresentam bastante complexas na dinâmica psicossomática. Essa modalidade prioriza o atendimento humanizado, enfatizando a integração dos aspectos biopsicossociais, ecológicos e espirituais. $^{(23)}$

As intervenções propostas não têm como objetivo erradicar a dor, mas reduzir sua intensidade, o desconforto que a acompanha, a preocupação na manutenção do desempenho na realização de tarefas, a diminuição do uso de medicamentos, o controle dos sintomas associados, ajustes comportamentais, desenvolvimento de estratégias de enfrentamento da dor, reconstrução da auto-imagem e resgate da autoconfiança. ${ }^{(21,24)}$

Nesse contexto, considerando que a dor crônica exige 
cuidados constantes, um aspecto que merece atenção é a participação do próprio paciente como agente de seu tratamento, colaborando no processo de busca por melhores condições de saúde e qualidade de vida.

Essas observações nos levam a propor o uso da eutonia nessa síndrome, já que essa técnica contempla em sua execução a necessidade do autoconhecimento e a participação ativa do paciente como seu próprio curador. Ela é uma prática orientada à consciência e integração corporal através do equilíbrio harmonioso do tônus. Traz à consciência o funcionamento do corpo: padrões habituais de postura, de movimento, modo de distribuição das tensões, alinhamento e/ou desvios do eixo físico, orientando a construção de um centro de equilíbrio, no qual o indivíduo tem a possibilidade de se relacionar melhor com o mundo e com ele mesmo. ${ }^{(12,13,14,25)}$

Ao ampliar sensitivamente a consciência corporal, encontra-se gradativamente o tônus mais adequado à cada atividade da vida diária. Este tônus muscular é acompanhado por um tônus psíquico (psicotônus) que é a mudança emocional em conseqüência das atividades de seu cotidiano e da sua história de vida. ${ }^{(13,14,25)}$

O objetivo básico da eutonia é propiciar ao ser humano a capacidade de aprender a se observar, registrar o fenômeno corporal no momento em que ocorre, estimular a sensibilidade quebrando modelos posturais que dificultam a flexibilidade do tônus, incentivar a disposição para

\section{REFERÊNCIAS}

1. Yunus MB: Fibromyalgia syndrome: clinical features and spectrum. J Musculoskeletal Pain. 2: 5-18,1994.

2. Wolfe F, Smythe HA, Yunus MB et al: The American College of Rheumatology 1990

Criteria for the Classification of Fibromyalgia. Arthritis Rheum 33: 160-72, 1990.

3. Atra E, Pollak DF, Martinez JE: Fibromialgia: etiopatogenia e terapêutica. Rev Bras Reumatol 33: 65-72,1993.

4. Angelotti GS: Fibromialgia: avaliação dos aspectos cognitivos, psicológicos e comportamentais. Campinas (SP), Pontifícia Universidade Católica de Campinas, Dissertação de Mestrado, 1999.

5. Ferraccioli G, Ghirelli L, Scita F. et al: Biofeedback training in fibromyalgia syndrome. J Rheumatol 14: 820-25, 1987.

6. Turner JA, Romano JM: Cognitive-behavioral therapy. In: BONICA J (ed.) The Management of pain. Philadelphia/ London: Lea \& Febiger, 1990.

7. Sousa MF: A Ferida Exposta: um estudo sobre a auto-imagem de crianças com lesões corporais. São Paulo: Psicologia Clínica da Pontifícia Universidade Católica de São Paulo, Dissertação a investigação, para a experimentação e para a ousadia de vivenciar algo novo.

Os resultados desse estudo mostram uma melhora significativa na intensidade da dor durante o período da aplicação da eutonia. Outro dado relevante é a manutenção dessa melhora no ano subseqüente ao tratamento. A melhora da dor tem sido observada em outros estudos psicológicos, em especial na terapia cognitivo-comportamental. Porém, chama a atenção a longa duração do efeito dessa proposta terapêutica. ${ }^{(22)}$

Em relação ao impacto na qualidade de vida, este foi bastante significativo, embora em menor intensidade após um ano da intervenção. O impacto positivo atingiu a maior parte das escalas do SF-36, sugerindo que seu efeito não pode ser atribuído totalmente à melhora da dor.

O estudo mostra que essa técnica psicológica é útil no tratamento da FM. Não houve modificação na rotina terapêutica anterior dos pacientes estudados.

Conclui-se, portanto, que a eutonia é útil como método terapêutico na FM, nesse estudo associado ao tratamento habitual dos pacientes avaliados. Esse efeito é particularmente importante na intensidade da dor difusa crônica e da qualidade de vida. O próximo passo é realizar estudos controlados para verificar a intensidade da influência da eutonia em relação às medidas educativas e exercícios físicos e estudos de natureza qualitativa, para se entender os mecanismos pelos quais essa técnica atua.

de Mestrado, 2001.

8. Nielson WR, Walker C, McCain GA: Cognitive behavioural treatment of fibromyalgia syndrome: preliminary findings. J Rheumat 19: 98-103, 1992.

9. Keel PJ, Bodoky C, Gerhard U, Müller : Comparison of Integrated Group Therapy and Group Relaxation Training for Fibromyalgia. The Clinical Journal of Pain 14: 232-8, 1998.

10. Portnoi AG: Dor, stress e coping: Grupos Operativos em doentes com síndrome de Fibromialgia. São Paulo: Instituto de Psicologia da USP, Tese de Doutorado, 1999.

11. Brasio KM, Laloni DT, Fernandes QP, Bezerra TL: Comparação entre três técnicas de intervenção psicológica para tratamento da fibromialgia: treino de controle de stress, relaxamento progressivo e reestruturação cognitiva. Rev Ciências Medicas 12: 307-318, 2003.

12. Alexander G: Eutonia: um caminho para a percepção corporal. Editora Martins Fontes, São Paulo, 1983.

13. Brighel-Müller GE, Winklera: Pedagogia e terapia em eutonia: guia de regulação psicotônica. Editora Summus, São Paulo, 1999.

14. Dascal M: Eutonia: o saber do corpo. Campinas, Instituto de Artes da UNICAMP, Dissertação, 2005. 
15. Jensen MP, Karoly P: Self-report scales and procedures for assessing pain in adults. In: Turk DC \& Melzack R. Handbook of Pain Assessment, $3^{\text {th }}$ ed, 135-51, 1992.

16. Ciconelli RM: Tradução para o português e validação do questionário genérico de avaliação de qualidade de vida Medical Outcomes Study 36-item Short Form Health Survey (SF-36). São Paulo, Escola Paulista de Medicina, Tese de Doutorado, 1997.

17. Alarcón GS, Bradley LA: Advances in the treatment of fibromyalgia: current status and future directions. Am J Med Sci 315: 397- 404, 1998.

18. Bennett RMA: Multidisciplinary approach to treating fibromyalgia In: Vaeroy $\mathrm{H}$ \& Merksey $\mathrm{H}$ : Progress in Fibromyalgia and Myofascial Pain, p. 393-410 Amsterdam, Elsevier, 1993.

19. Bennett RM, Buckhardt CS, Clark SR, O’Reilly C, Wiens AN, Campbell SM: Group treatment of fibromyalgia: a six month outpatient program. J Rheumatol 23: 521-8, 1996.
20. Gentry WD \& Owens D: Pain groups In: Holzman AD, Turk DC: Pain management: a handbook of psychologial treatment approaches. New York: Pergamon,100-12, 1986.

21. Beck AT, Rush AJ, Shaw BF, Emery G: Terapia cognitiva da depressão. Rio de Janeiro, Editora, Zahar, 1982.

22. Rangé B: Psicoterapias cognitivo-comportamentais: um diálogo com a psiquiatria. Porto Alegre, Editora Artmed, 2001.

23. Lipowiski ZJ: What does word psychosomatic really mean? A historical and semantic inquiry. Psychosomatic Medicine 46: 153-171,1984.

24. Ferreira EAG, Marques AP, Matsutani LA, Vasconcellos EG, Mendonça LLF: Avaliação da dor e estresse em pacientes com fibromialgia. Rev Bras Reumatol 42: 104-110, 2002.

25. Maeda CL: A Eutonia e o processo de individuação: uma abordagem psicossomática. São Paulo: Psicologia Clínica da Pontifícia Universidade Católica de São Paulo, Dissertação de Mestrado, 2000 